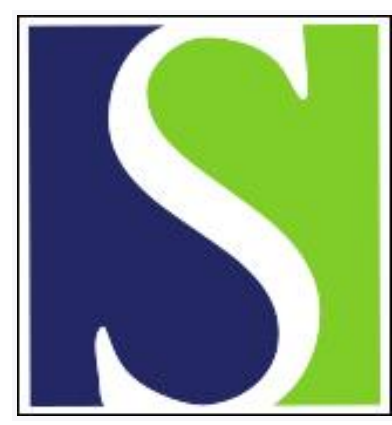

Scand J Work Environ Health 1996;22(6):405-414

https://doi.org/10.5271/sjweh.161

Issue date: Dec 1996

Quantification of historical exposures in occupational cohort studies

by Stewart PA, Lees PSJ, Francis M

Key terms: cohort study; epidemiologic study; exposure assessment

This article in PubMed: www.ncbi.nlm.nih.gov/pubmed/9000307

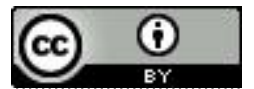




\title{
Quantification of historical exposures in occupational cohort studies
}

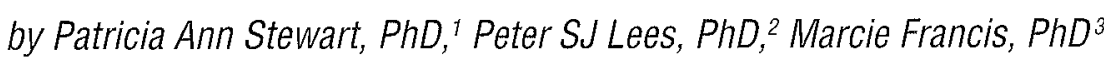

\begin{abstract}
Stewart PA, Lees PSJ, Francis M. Quantification of historical exposures in occupational cohort studies. Scand J Work Environ Health 1996:22:405-14.

Relatively few investigators have estimated quantitative exposure levels in epidemiologic studies, and, for those that have, few have discussed the strengths and weaknesses of their estimation method with respect to other methods. This paper reviews the steps for developing quantitative exposure estimates that have been used in published studies. First, the qualitative considerations to be evaluated in the selection of the agent to be estimated (ie, the disease mechanism, the effects of exposure mixtures and interactions, the physical state of the agent, and the routes of exposures) are discussed. Considerations for developing exposure groups are then presented, including work history and exposure information characteristics, the homogeneity of exposures within exposure groups, the exposure estimation method, and the disease risk analyses to be performed. The various exposure estimation approaches are reviewed for their strengths and weaknesses, including the calculation of the mean exposures from existing exposure measurements, statistical models, measurement data from surrogate exposures, and professional judgment. Recommendations for future studies are provided.
\end{abstract}

Key terms cohort studies, epidemiologic studies, exposure assessment.

One of the key criteria in the establishment of causal links between occupational hazards and disease in epidemiologic studies is the existence of a dose-response association (1). Measurements of dose (internal concentrations of an agent) are rarely ever available, so that the concept of exposure (external concentrations) has been substituted for that of dose. Exposure measurements are much more common than dose measurements, but they are seldom available except for more recent years. Because occupational exposures are difficult to assess without measurements, surrogate measures, such as ever or never employed, duration of employment, and qualitative or semi-quantitative estimates, have typically been used to investigate exposure-response associations. The limitations of these approaches have been described elsewhere (2).

In spite of the crudeness of these approaches and the inherent exposure misclassification that undoubtedly accompanies them, these approaches have been successful in observing associations and establishing causality. The environment in which epidemiologic research on chronic occupational hazards has been conducted has changed, however, over the last 20 years. First, there is an in- creased emphasis, both in the private sector and in regulatory agencies, on quantitative risk assessment for evaluating the potential relationship between exposure and health effects. A second change is that relative risks arising from exposures of concern today may be smaller than the risks from exposures (eg, asbestos) evaluated historically (3). For diseases with large relative risks, excesses can be observed even when severe misclassification occurs (such as what might occur using evernever and duration analyses). However, the impact of misclassification on investigations of diseases with lower risks may cause associations to be missed unless the misclassification is diminished by better exposure estimation. Third, the mechanisms of disease for the exposure being investigated is usually not well understood and may not be as straightforward as simply accumulating exposure over subjects' lifetimes. In such situations, the exploration of different exposure measures may be useful (4), but the absence of quantitative exposure estimates makes such an exploration difficult. A fourth change in the environment of occupational health research in the United States is the increased practice of monitoring airborne and, to a more limited extent, der-

1 Occupational Epidemiology Branch, National Cancer Institute, Rockville, Maryland, United States.

2 Johns Hopkins University, School of Hygiene and Public Health, Baltimore, Maryland, United States.

3 Formerly of Johns Hopkins University, now at TAS, Inc, Washington, DC, United States.

Reprint requests to: Dr PA Stewart, Occupational Epidemiology Branch, National Cancer Institute, EPN 418, Rockville, MD 20892, USA. 
mal exposures. Although it is unlikely that there will ever be enough appropriate monitoring data to develop personal exposure estimates for each worker for all years of a disease with a long latency, for recent years there are often sufficient numbers of monitoring results with which to estimate exposures. The availability of these data facilitates the development of exposure estimates and can increase the likelihood of finding an association over the more traditional methods (5).

As a result of these changes in the research environment, interest in, and emphasis on, the development of quantitative exposure estimates in occupational cohort studies has increased (6). The procedures to develop such estimates have not been standardized, however. Although this lack is likely to be due, in part, to the relative newness of the exposure assessment field, it is also a reflection of differences in the availability of monitoring results and other exposure data from study to study. The goal of this paper is to review the steps of the quantitative exposure assessment process with an occupational cohort study design. Quantitative assessment is defined as developing exposure estimates with units of measurement, such as parts per million, and not scores or evaluations such as low, medium and high.

First, some considerations for the identification of the exposure agent of interest are described. These include disease mechanisms, the effect of exposure mixtures and interactions, the physical state of the agent, and the routes of exposures. Considerations for forming exposure groups are then discussed, including work history and exposure information characteristics, the homogeneity of exposures within exposure groups, the estimation method and the disease risk analyses to be performed. Finally, quantitative assessment methods used to develop historical exposure estimates in the occupational cohort study design are reviewed. These methods include the use of measurement data of the agent of interest, statistical models, measurement data from surrogate exposure agents, and professional judgment. Recommendations for future studies are provided.

\section{Some qualitative considerations}

Identifying the exposure of interest in some studies may be straightforward. In others, however, it may be difficult, because it is not always immediately obvious what exposure agent should be assessed; choice of the wrong agent can result in an association remaining unobserved. First the agent assessed should be one for which a biologically plausible mechanism is expected from toxicologic studies. For example, the investigation of laryngeal and lung cancer led investigators to evaluate the size of machining fluid aerosols (7) and man-made mineral fibers (8), respectively. Mixtures of agents can increase the complexity of the exposure assessment ( 9 ) because it may not be clear which agent in the mixture is the causative agent or, alternatively, there may be several causative agents in the mixture. Complicating this issue is the fact that the agent of interest may be difficult or impossible to analyze. Therefore evaluating a surrogate exposure agent may be more feasible. For example, hexavalent chromium is generally used as the marker for a variety of chromium compounds even though they have widely varying toxicities.

Second, the exposure agent can be present in several physical states. If the agent is in the gaseous or vapor state, particulates are not present and dermal and ingestion routes are not important, an evaluation of the gas or vapor is sufficient. If particulates are also present, however, the gas or vapor can be adsorbed onto the particulate or it may off gas from the particulate. For example, in a study of formaldehyde workers, formaldehyde, formaldehyde-releasing particulates, and other particulates were present simultaneously in several of the companies under study (10). The authors did not find a risk for nasopharyngeal cancer from exposure to formaldehyde except when it was associated with particulates (11). Finally, it is important to evaluate exposure from all routes of exposure to determine their importance to total dose. In the case of pesticides, for example, dermal exposure, not airborne exposure, is usually the primary route of exposure.

\section{Formation of exposure groups}

The underlying goal of exposure assessment is to develop estimates of exposure for each agent of interest. We use the term "exposure estimation class" to describe the administrative or environmental class for which investigators develop exposure estimates. Historically, in cohort studies, a plant or department was used as the exposure estimation class. More recently, the job has become the typical exposure estimation class. Ideally, however, the exposure estimation would have made for the individual study subject. Because measurement data are not usually available for most persons over the study period, however, and because it is usually not practical to develop exposure estimates for individual subjects even if they are available, investigators develop exposure groups of persons with similar exposures.

The term homogeneous exposure group has been recently popularized to identify groups of persons who experience exposures similar enough that monitoring of any worker in the group provides data useful for predicting the exposure of the remaining workers (12). This definition is often interpreted to mean that workers 
holding the same job are in the same homogeneous exposure group (13). The variability of exposures within a job, however, may be greater than traditionally recognized (14), and so for this reason the term exposure group rather than homogeneous exposure group has been used in this report to describe the estimation class.

The exposure estimation class selected depends on the particular characteristics of the data. The detail of the records by which study subjects are linked with task assignments (eg, personnel records) and by which tasks are related to exposure (eg, industrial hygiene records) influence how specific the exposure estimation class can be. In many cohort studies employer personnel records are used to identify subjects by job title, department, and year, and exposure levels are calculated or inferred for job-department-year combinations. Where the records lack information on job tasks, however, it may be necessary to select a different exposure estimation class (table 1). For example, in a study of miners, the personnel records did not identify the section of the mine in which study subjects worked and some records did not identify the job title or the mine (15). Mine section was therefore ignored, and the subjects were grouped by occupational group and mine, mine where the job title information was missing, and year where both job title and mine information were missing.

The second consideration in selecting the exposure estimation class is the availability of exposure information. Exposure information may be quantitative (eg, measurement data) or descriptive (eg, job descriptions). In general, if only measurement data are used for estimating exposures, the exposure estimation class is likely to be less homogeneous with regard to the exposure level than if other data are also used. In a study of man-made mineral fiber workers, for example, measurement data were so limited that all jobs within a facility were grouped and given the same exposure mean (16), and therefore distinction could not be made between higher and lower exposed workers.

The exposure estimation method can also affect the selection of the class of estimation. The class must gen- erally be based on broader categories if sole reliance is placed on exposure measurement data because the number of measurements is usually limited. For example, only eight job categories were developed in a study that used regression analysis to predict exposure levels of ethylene oxide workers (17). Alternatively, if qualitative information is used to supplement measurement data, a more specific level of estimation may be possible, and, potentially, the homogeneity of the exposure within each group is increased.

An evaluation of the homogeneity of exposures for persons within an exposure group can be examined when sufficient measurement data are available. For example, in a study of styrene users, investigators conducted a variance components analysis and determined that the variation of the exposure measurements could be attributed to the sampling date and to company and individual variation (18). Little exposure variation among job titles was found, however, after product, process and type of exposure (direct or indirect) were accounted for. Consequently, job title was ignored and risk estimates were developed for combinations of product, process and exposure type. Where measurement data are not available, a careful examination of the tasks, locations, and time spent at the tasks and locations can help to determine if these characteristics are likely to result in different exposures. If it is found that the members of a presumed exposure group are actually not homogeneously exposed, that is, that the exposure means are very different, formation of different exposure groups may be necessary. For example, in a study of acrylonitrile workers, variation in exposures was considerable among engineers in several of the plants in the study because the time the engineers spent in the acrylonitrile unit varied from $0 \%$ to $100 \%$. Assigning the same exposure estimate to all engineers in these plants would introduce substantial misclassification to the exposure estimates. One of the authors (PAS) therefore created several exposure groups of engineers based on the differences of time in the acrylonitrile unit. Another consideration when developing exposure groups is that persons within an exposure group must be affected similarly by changes in the work-

Table 1. Examples of exposure estimation classes.

\begin{tabular}{|c|c|c|}
\hline Reference & Exposure estimation class & Influencing factors \\
\hline Rice et al (27) & Task-product & $\begin{array}{l}\text { Large number of facilities } \\
\text { Few measurements relative to number of facilities and tasks monitored }\end{array}$ \\
\hline Hornung et al (17) & Job-Iocation & $\begin{array}{l}\text { Limited measurement data } \\
\text { Method of estimation }\end{array}$ \\
\hline Seixas et al (15) & $\begin{array}{l}\text { Occupational group-mine-year } \\
\text { Mine-year, year }\end{array}$ & $\begin{array}{l}\text { Section of mine not identified in personnel records or } \\
\text { measurement data } \\
\text { Job title, mine often missing }\end{array}$ \\
\hline Lemasters et al (18) & Product-process-exposure type & Limited measurement data \\
\hline Dement et al (29) & Uniform task-exposure zones & Short-term task and area measurement data \\
\hline Dodgson et al (16) & Facility-year & Limited measurement data \\
\hline
\end{tabular}


Table 2. Quantitative exposure estimation approaches described in occupational epidemiologic papers published in 1985-1995.

\begin{tabular}{lcc}
\hline Approaches & Number & Percent \\
\hline Calculation of means & 25 & 35 \\
Calculation of time-weighted averages & 5 & 7 \\
Interpolation & 2 & 3 \\
Statistical models & 4 & 5 \\
Surrogate exposures & 2 & 3 \\
Professional judgment & 34 & 47 \\
\hline Total & 72 & 100 \\
\hline
\end{tabular}

place so that the homogeneity of exposures is maintained over time.

Individuals within an exposure group must also be similar for all exposure measures being evaluated. Thus, if disease risks are to be evaluated for both peak and 8-h time-weighted average (TWA) exposures, either all people assigned to an exposure group must have the same peak levels and the same TWA levels or two sets of exposure groups will be necessary. The latter situation may be more typical because different exposure measures are not necessarily correlated. In a study of formaldehyde workers, for example, the correlation between different measures of exposure was evaluated and found to vary widely from plant to plant (19). For peaks and 8-h TWA values the overall correlation for all the plants was 0.5 , but it ranged from 0.1 to 0.8 across the 10 plants in the study. Thus developing exposure groups for one exposure measure and using them for a second could cause enough misclassification to obscure an association with the disease of interest. As interest in other exposure measures increases, more precise exposure groups or more sets of exposure groups become necessary. Control for confounding occupational exposures or evaluating disease risks for several exposures increases the complexity of developing exposure groups, as persons within each exposure group must be homogeneous with respect to these exposures also.

\section{Development of quantitative exposure estimates}

After exposure groups are developed, the next step is to set up exposure estimates. We reviewed occupational cohort studies published over the last 11 years (19851995) in six journals that focus on occupational exposures and disease to determine what estimation techniques have been used (American Industrial Hygiene Association Journal, American Journal of Industrial Medicine, Annals of Occupational Hygiene, Applied Occupational and Environmental Hygiene, British Journal of Industrial Medicine, and Scandinavian Journal of Work, Environment \& Health). The use of quantitative exposure estimates was indicated in 72 epidemiologic studies or methods papers. In general, three approaches to the estimation of exposure levels were used (table 2). One-third of the papers $(\mathrm{N}=25)$ indicated that the primary estimation technique was the calculation of mean exposures from monitoring results, and almost half $(\mathrm{N}=34)$ described a professional judgment approach. In the remaining studies the primary estimation technique was the calculation of TWA values from area or short duration measurements weighted by time, the interpolation of exposure from measurement data, or the use of statistical models or surrogate exposure measurements to predict exposures.

For the purposes of this review, these estimation approaches can be grouped into arithmetic calculations, prediction models, and professional judgment.

\section{Arithmetic approaches}

Arithmetic calculations include (i) the calculation of means directly from measurements, (ii) the calculation of TWA values using short duration or area measurements weighted by time, and (iii) interpolation of measurement data.

Calculation of means from measurement data. Full-shift, personal measurement data are the best resource for developing quantitative exposure estimates of 8 -h TWA values. When such measurements are available over most of the jobs and years of the study, arithmetic or geometric means can be used as the estimates of exposure. Arithmetic means have been suggested as being preferable when cumulative exposure is being estimated because cumulative exposure assumes a linear exposureresponse model (ie, that every molecule counts equally) (20). Simple means or means of means have been used as estimates of exposure. Calculating means is attractive because it is simple, reproducible, and requires few assumptions. This approach requires, however, sufficient monitoring results across jobs and over the study period to complete all or most of the exposure group-year combinations, or "cells," needing estimation. Because this abundance of data does not usually occur in studies of diseases with long latency, total reliance on means calculated from full-shift, personal monitoring data is usually not possible.

The monitoring results used to estimate historical exposures can be current, historical, or both, but measurements made during the period being estimated are obviously preferable. If historical changes in the operation were likely to have had a minor effect on exposures, the use of current data to develop historical exposure estimates is probably acceptable. Current monitoring data can also provide information on jobs that have never been monitored to facilitate the assignment of their relative exposure ranking among the study jobs. In addition, current data can be used as the reference 
standard for comparing measurement data across many work sites.

The primary concern with sole reliance on measurement data is the question of representativeness of the data to the true long-term exposure. (Long-term is used in this respect to represent the period of time represented by an exposure estimate. Typically, this is a year.) Much monitoring has been conducted for purposes of compliance or control evaluation $(21,22)$ and popular belief is that these types of data may be biased, because the focus of such measurements is on highly exposed people or conditions (23). How this type of monitoring affects the magnitude of the mean and whether it also affects the relative ranking of jobs is unclear at this time.

Another reason measurement data may be unrepresentative of long-term exposures is that they have usually been collected under nonrandom sampling conditions (eg, measurements may have been made during unexpected occurrences, eg, a spill) or under planned conditions that occur periodically (eg, an operating unit shut down for annual maintenance). If the fraction of the monitoring results for these conditions is disproportionate to the actual frequency of occurrence, the calculated mean estimate would be distorted toward the unusual conditions. For example, if only two measurements are available for a given job, one taken under normal production conditions and the other when the process was down, a situation that occurs one day per month, the mean of these two results will be biased towards the shutdown exposure, even though each represents a normal and true exposure condition.

If the measurement data identify the conditions of sampling, investigators may be able to estimate the frequency of the condition and thereby weight the measurements of each condition accordingly to develop an overall annual weighted mean exposure. This has been the approach taken for shutdowns (24) and season or temperature differences $(7,25,26)$. Another cause of unrepresentativeness to long-term means is the use of a sampling or analytic method that produces biased results. Some investigators have adjusted results of biased methods (27), while others have excluded them (17). If the variability of the differences between the biased and unbiased method is high, it may be best to exclude them. A low variability, however, suggests that the bias may be systematic, and therefore adjusting the results would be acceptable.

Calculations using area or short-duration measurement data weighted by time. Many historical data are area measurements or personal measurements of short duration. The use of these data as surrogates for full-shift personal samples can be problematic if they are not representative of full-shift personal exposures (28). To make them more representative, some investigators have weighted area and short-term monitoring results by time. For example, in a study of workers in an asbestos textile company, the authors had area and short-term task measurements of asbestos (29). Eight-hour TWA values were calculated for each job by multiplying the area measurements by the time spent in each area, adding an increment from the task measurements where appropriate, and summing the products. If area or short-duration measurements are used, their validity can be assessed by comparing them with full-shift, personal monitoring data, such as was done in a study of aluminum workers (30), in which the authors found differences among the measurements and therefore made adjustments.

When estimates of time are used to weight the exposure concentrations, error arises from the estimates of time in addition to the measurement data. Little information is available on this latter type of error or even on the variability of time spent performing tasks. Recently, in a study of telephone linemen, the mean observed worktime (in hours) was found to have standard deviations of $13 \%$ to $31 \%$ for five job groups (31).

Interpolation. Interpolating exposure estimates from measurement data has been used to estimate exposures for years where data are nonexistent. This approach is simple and reproducible, but its validity is less apparent. The implicit assumption of such an approach is that exposure levels change gradually over time, for example, from a gradual increasing awareness of health hazards over time or a gradual decline in the efficiency of a ventilation system. In many cases, however, changes in exposure are more abrupt because they result from a change in the process or installation of engineering controls. Another disadvantage of interpolation is that it requires data in the early years of a study, although this is often the period when data are very sparse or missing.

\section{Prediction methods}

Predictions using statistical models. When monitoring data are available for some exposure group-year cells but are missing for others, statistical models can sometimes be used to develop exposure estimates. An analysis of variance (ANOVA) was used, for example, to complete missing cells in a granite shed study of silica-exposed workers (25), and regression analysis was used in another study to predict exposure levels to ethylene oxide (17). In this latter study, several production variables, such as various engineering controls, size of the sterilant chamber and pounds of ethylene oxide used per year, were examined for their significance in a regression model using measurement means as the dependent variable. Seven variables and two interaction terms were identified as significant. These nine terms, which explained $90 \%$ of the variation, were then used in a prediction model. 
The approach used by Hornung et al (17) is deterministic in that the authors identified a priori parameters based on physical and chemical principles to predict exposures. Yu et al (32) recommended that the exposure assessment process be deterministic in approach and suggested that changes in the workplace can produce either incremental (additive, eg, start up of a new process) or ratio (multiplicative, eg, installation of local exhaust ventilation) changes in exposure concentrations. They evaluated the advantages and disadvantages of a deterministic statistical model using a simple linear regression model, a multiple linear regression model, and a weighted least squares model with step functions. The estimates of exposure derived from the least squares method were closest to the reference monitoring results.

Statistical modeling has the advantage of developing unbiased estimates in a fairly reproducible way, but, although powerful, it has its limitations. First, models need a small number of variables (eg, exposure groups and engineering controls) relative to the number of means calculated from the measurement data (table 3). Typically, however, a large employer will have several hundreds to thousands of job titles (33), of which few have been monitored. Grouping of jobs into a relatively small number of exposure groups may be necessary before a statistical model can be used, but it may be inappropriate due to the heterogeneity of exposures within the exposure groups. A second problem of such models is that they require some measurement data for all conditions and situations being predicted. For example, measurements are necessary for years both before and after the installation of controls. If monitoring data are not available for particular environmental conditions (either in time or for a process or department), a statistical model cannot be used to predict the exposures.
Prediction of surrogate exposures from monitoring data. Esmen (34) suggested that when historical monitoring data exist for one agent, the data can be used to predict the exposures of the second agent if the two agents are present in the same relative concentrations throughout the process. This approach was used to estimate the benzo[a]pyrene exposures of aluminum smelting workers simultaneously exposed to benzene-soluble materials (30). In that study, although there were measurements for both benzene-soluble material and benzo[a]pyrene exposures after 1976, prior to 1976 there were only benzene-soluble material measurements. The authors therefore derived a ratio of benzo[a]pyrene:benzene-soluble material measurements from 1976 to 1983 for occupational groups and, assuming the ratio remained the same, applied this ratio to pre-1976 benzene soluble material levels. This is a simple and reproducible method of predicting exposure. A careful evaluation must be made, however, to ensure that process or control changes did not differentially affect the exposures to the two agents of interest, either within a job or across jobs over time. This evaluation would be done by identifying the tasks of the jobs and determining how these tasks interact with the source being changed, and it would include consideration of the distance to and time spent in contact with the agents of interest as they changed. Local changes, for example, installation of a sampling cabinet, would be likely to have more of a differential effect than area changes, for example, installation of a general dilution fan.

\section{Professional judgment}

The approaches described may not be possible in many studies, but quantitative exposure estimation may still be desired. As a result, investigators frequently indicate that "professional judgment" was used to develop quantita-

Table 3. Examples of statistical models used to estimate exposures. (NP = not provided)

\begin{tabular}{|c|c|c|c|c|}
\hline Reference & Technique & $\begin{array}{l}\text { Number of } \\
\text { measurements }\end{array}$ & $\begin{array}{c}\text { Number of } \\
\text { exposure } \\
\text { groups }\end{array}$ & Variables \\
\hline Woskie et al (26) & Analysis of variance & 534 & 5 & $\begin{array}{l}\text { Climate } \\
\text { Facility } \\
\text { Distribution of workers }\end{array}$ \\
\hline Hornung et al (17) & Multiple linear regression & 2350 & 8 & $\begin{array}{l}\text { Product type } \\
\text { Age of product } \\
\text { Calendar year } \\
\text { Type of exhaust } \\
\text { Aeration procedure } \\
\text { Sterilizer volume }\end{array}$ \\
\hline Dement et al (29) & Multiple linear regression & 5952 & 9 zones & $\begin{array}{l}\text { Process, control changes } \\
\text { Time periods }\end{array}$ \\
\hline Hallock et al (7) & Analysis of covariance & 394 & NP & $\begin{array}{l}\text { Facility } \\
\text { Machining fluid } \\
\text { Operation } \\
\text { Date }\end{array}$ \\
\hline Eisen et al (25) & Analysis of variance & 1153 & 25 & $\begin{array}{l}\text { Facility } \\
\text { Season } \\
\text { Year }\end{array}$ \\
\hline
\end{tabular}


tive estimates (ie, incorporating qualitative information into the exposure estimate). This approach is usually deterministic in nature. A study of formaldehyde workers can serve as an example of this approach (35). First, a limited number of arithmetic mean exposures were calculated from the monitoring results (33). To develop exposure estimates for jobs that had not been monitored, the investigators modified the exposure concentrations of jobs with similar tasks that had been monitored. Historical exposures were estimated by modifying more recent exposure estimates by amounts that reflected the effect that changes in the workplace were likely to have had on the exposure levels.

The limitation of studies using this approach is that the methods used to develop the exposure estimates are usually not explicitly described. This lack of explicitness makes the evaluation of the exposure estimates difficult for readers, and it reduces the reproducibility of the estimates. Some investigators have, however, provided detailed descriptions of the assessment procedures. A study of workers in 10 rock wool insulation production facilities used an approach similar to that used in a formaldehyde study (33) to estimate historical exposure levels, but the effects resulting from the changes were explicitly described (16). The effects of two of the most significant process changes, the addition of oil to the fiber, and a change in the diameter of the fiber were estimated after fiber concentrations were generated in an experimental chamber under varying conditions. Other changes in the workplace were also described, and multipliers estimating their effect were reported. To develop the historical estimates, the appropriate multiplier for each change was applied to the mean of the monitoring results to derive the estimated exposure level for years when monitoring results were nonexistent.

In another study several estimation methods were used (24). Because the exposure measurement data and qualitative information varied from job to job and year to year, no single estimation method could be used for all the missing cells without increasing misclassification. The approach was therefore to use the best method possible for each exposure group-year cell, to have defined criteria and a hierarchy for using each method, and to retain a description of the assumptions made with each estimate. Means of monitoring results and TWA values based on time-weighted area, short duration, or personal measurements of other jobs were calculated. Historical exposures were estimated by identifying changes that affected exposure levels. An estimate of the effect of these changes and an estimate of the percentage that the source being changed contributed to the overall emissions were applied to the exposure estimates already developed for an adjacent time period. Using professional judgment in combination with the measurements, qualitative information and defined criteria gave the in- vestigators the flexibility to maximize the use of the available data while developing reproducible and reasonable estimates.

\section{Discussion and recommendations}

Investigators have taken a variety of approaches to develop quantitative retrospective occupational exposure estimates for epidemiologic studies, and enough reports are now in the literature to compare the estimation approaches and to make recommendations.

The first step in the development of estimates is to identify the appropriate agent of exposure. Historically, investigators have taken a simplistic approach to this step, but it is increasingly recognized that such an approach may be undesirable since it can result in either the investigation being left without sufficient information to comment on the true exposure-response relationship or speculation about the true relationship after the study is completed. Neither option is preferable. We recommend that considerations be made as to the sizes of particulates, the presence of mixtures and interactions, and the effect of physical state and routes of exposures in each study. In addition the manner in which the exposure assessment process reflects these variables should receive attention, where appropriate.

Development of exposure groups is a critical step in most studies. Work history records may be insufficient to distinguish among varying exposure environments of the jobs. In other studies there may be insufficient monitoring data. When confronted with missing information, investigators have the option to group jobs into very broad exposure categories (whether within a facility or across facilities) to allow sole reliance on measurement data or they can incorporate qualitative data if they wish to keep jobs separate and distinct. In the first case, professional judgment is required to group a larger number of jobs into a smaller number of exposure groups, because an exposure level is implicitly being assigned to the jobs without data. Although this approach makes the assessment effort more manageable, it could result in jobs being inappropriately categorized together (ie, jobs that have different mean exposures, different variability of exposures, or different confounding exposures can be grouped).

In the second approach, maintaining a large number of jobs probably means that professional judgment will be necessary to estimate exposure levels for many jobs. This process is more tedious and resource intensive than the first. If the exposure environment is well characterized and rigorous assessment procedures are made explicit and followed, it may result in more accurate exposure estimates than developing relatively few expo- 
sure groups does because categorical analyses have lower power than continuous data analyses (36). Without rigorous procedures and careful evaluation, however, the approach may be more subjective and, therefore, less accurate and also more open to criticism.

Rarely have study investigators described their approach or justification for developing exposure groups. An exception to this lack of information was a study of electrical workers (37), in which a detailed description was provided as to how the investigators developed their exposure groups. We recommend that the exposure estimation class used in studies be identified and documented in a similar manner.

All of the exposure estimation approaches described are dependent on the existence of at least some measurement data. Without measurement data either from the workplace under study or from similar workplaces, quantitative exposure estimates should probably not be attempted (38). The studies cited generally provided some detail as to what measurement data were available for exposure assessment, but, in most cases, more information would have been helpful for understanding the data. For example, in some reports, the number of results, the type (personal or area), and the duration of the measurements was not provided. A description of the distribution of monitoring results over the study years and over the jobs, departments, or facilities would be useful.

Historical monitoring data are usually sparse. Even where available, they often do not represent full-shift personal exposures because the data were collected to evaluate compliance with regulatory standards, to evaluate controls, or to assess exposure levels during unusual conditions or because the sampling technology did not permit such evaluations. If the measurements were taken for expediency, however, they may be comparable to full-shift exposures (39). For other purposes, the industrial hygiene community has not yet developed a satisfactory method for adjusting such measurements to longterm TWA values, and more research needs to be done in this area. At a minimum, it would be helpful to understand the conditions that exist when such measurements are and are not representative of full-shift personal exposures and how they can be handled (22). For example, when exposure concentrations are homogeneous in an area (ie, there are multiple small sources of a gas or vapor or well-mixed air in a room), area measurements may be an acceptable alternative to personal measurements. If an exposure arises primarily from a task, however, area measurements are less likely to be representative of personal exposures (28). Investigators may also delete or weight differentially the measurement data. When this is the case, the conditions when area or shortterm measurements were used should be characterized, and the criteria for deleting or weighing any measurement should be identified.
As has already been indicated, the use of professional judgment, although perhaps the weakest of the estimation approaches with regard to statistical validity and reliability, is, nonetheless, the second most common approach in developing quantitative estimates. In a sense, however, professional judgment is used in all the estimation approaches. Developing exposure groups without measurement data requires professional judgment because exposure levels are implicitly estimated. Professional judgment is also required to determine what, if any, measurement data are to be discarded for lack of representativeness or due to a poor sampling and analytic method; what adjustments are required to ensure comparability among different exposure information sources, for example, measurement data from different monitoring and analytic techniques (27); and how and which data are to be weighted. Use of statistical models also requires professional judgment to develop the best model (17).

The use of professional judgment as an estimation approach has generally been characterized, however, as having vague descriptions of what and how information was used to develop the exposure estimates. This is understandable because retrospective exposure assessment is still fairly new, and quantitative information on how exposure variables affect exposure levels has not been generated. The professional judgment approach to exposure estimation is often justly criticized, however, as being the industrial hygienist's "black box," where the accuracy and reliability of such estimates are not known. More careful and detailed documentation is needed.

In an attempt to provide such detail in a study of acrylonitrile workers, an interactive computerized program was developed that documented what information was known about each job (eg, process description, tasks, location, etc) and what was assumed (40). Several estimation methods were used, and criteria for using the methods were identified (24). The estimation method and the data used to develop each estimate were also retained, and they provided a detailed description of how each exposure estimate was derived (41). Thus, even when professional judgment is used, rigorous procedures with extensive documentation can be provided. Without such documentation, the credibility of the study is diminished.

In addition to documentation of the exposure estimation process, the accuracy and reliability of the estimates should be evaluated, where possible, to quantify the likely degree of misclassification and its effect on disease risk estimates. This procedure has been carried out only in a few studies using air monitoring results as the reference standard. Estimates of ethylene oxide exposures developed by industry raters and estimates derived from a regression model were compared with a subset of the monitoring data reserved for this purpose (17). Several 
of the methods used in the study of acrylonitrile workers were also evaluated using a subset of the monitoring data (24). In the man-made mineral fibers study, exposure estimates were compared with air measurements generated from the reconstruction of historical conditions (16). Thus, currently, it may be possible to quantify the accuracy of each approach. Presumably, direct use of measurements and statistical approaches is better than the professional judgment techniques, but more data are needed to determine to what degree.

Although all of the approaches described for estimating historical exposures have limitations, it is important to emphasize that investigators conducting exposure assessments may be forced to develop estimates using less than desirable methods because of the lack of data and limited resources. It is likely that this need to "make do" increases misclassification. If nondifferential, this type of misclassification usually decreases the likelihood of finding an association if it exists (42). The likelihood of finding a false positive association is much lower. As long as the degree of misclassification is less than that observed for cruder measures of exposure (eg, duration of employment) quantitative estimates of exposure are preferable.

\section{Concluding remarks}

Historical exposure assessment requires an opportunistic approach, taking advantage of what information is available and developing creative and innovative approaches to exploit that information. It is important that details be provided as to the quality and quantity of the available data and that an explicit description of the estimation methods used be provided. In addition, an attempt should be made to validate the estimates wherever possible.

\section{References}

1. Monson RR. Occupational epidemiology. Boca Raton (FL): CRC Press, 1980:101.

2. Stewart PA, Herrick RF. Issues in performing retrospective exposure assessment. Appl Occup Environ Hyg 1991;6:4217.

3. Wegman D. Issues in the epidemiologic evaluation of exposure-effect relationships. In: Rappaport SM, Smith TJ, editors. Exposure assessment for epidemiology and hazard control. Chelsea (MI): Lewis Publishers, 1991:159_74.

4. Checkoway H, Rice CH. Time-weighted averages, peaks, and other indices of exposure in occupational epidemiology. Am J Ind Med 1992;21:25-33.

5. Blair A, Stewart PA. Do quantitative exposure assessments improve risk estimates in occupational studies of cancer? Am $J$ Ind Med 1992;21:53-63.

6. Herrick RF, Stewart PA. International workshop on retrospective exposure assessment for occupational epidemiologic studies. Appl Occup Environ Hyg 1991;6:417-20.

7. Hallock MF, Smith TJ, Woskie SR, Hammond SK. Estima- tion of historical exposures to machining fluids in the automotive industry. Am J Ind Med 1994;26:621—34.

8. Quinn MM, Smith TJ, Ellenbecker MJ, Wegman DH, Eisen EA. Biologically-based indices of fiber exposure for use in epidemiology. Occup Hyg 1996;3:103-11.

9. Hammond SK. The uses of markers to measure exposures to complex mixtures. In: Rappaport SM, Smith TJ, editors. Exposure assessment for epidemiology and hazard control. Chelsea (MI): Lewis Publishers, 1991:53—66.

10. Stewart PA, Cubit DA, Blair A, Spirtas R. Performance of two formaldehyde passive dosimeters. Appl Ind Hyg 1987;2:615.

11. Blair A, Stewart PA, Hoover RN, Fraumeni FJ Jr, Walrath J, O'Berg M, et al. Cancer of the nasopharynx and oropharynx and formaldehyde exposure. JNCI 1987;78:191—3.

12. Hawkins NC, Norwood SK, Rock JC, editors. A strategy for occupational exposure assessment. Fairfax (VA): American Industrial Hygiene Association, 1991:160.

13. Kromhout H, Heederik D. Occupational epidemiology in the rubber industry: implication of exposure variability. Am J Ind Med 1995;27:171-85.

14. Rappaport SM. Selection of the measures of exposure for epidemiology studies. Appl Occup Environ Hyg 1991;6:448 57.

15. Seixas NS, Moulton LH, Robins TG, Rice CH, Attfield MD, Zellers ET. Estimation of cumulative exposures for the National Study of Coal Workers' Pneumoconiosis. Appl Occup Environ Hyg 1991;6:1032-41.

16. Dodgson J, Cherrie J, Groat S. Estimates of past exposure to respirable man-made mineral fibers in the European insulation wool industry. Ann Occup Hyg 1987;31:567-82.

17. Hornung RW, Greife AL, Stayner LT, Steenland NK, Herrick RF, Elliot LJ, et al. Statistical model for predicting retrospective exposure to ethylene oxide in an occupational mortality study. Am J Ind Med 1994;25:825-36.

18. Lemasters GK, Carson A, Samuels SJ. Occupational styrene exposure for twelve product categories in the reinforced-plastics industry. Am Ind Hyg Assoc J 1985;46:434-41.

19. Blair A, Stewart PA. Correlation between different measures of occupational exposure to formaldehyde. Am J Epidemiol 1990;131:510-16.

20. Seixas NS, Robins TG, Moulton LH. The use of geometric and arithmetic mean exposure in occupational epidemiology. Am J Ind Med 1988;14:465-77.

21. Ulvarson U. Limitations to the use of employee exposure data on air contaminants in epidemiologic studies. Int Arch Occup Environ Health 1983;52:285-300.

22. Olsen E, Jensen B. On the concept of the "normal" day: quality control of occupational hygiene measurements. Appl Occup Environ Hyg 1994:9:245-55.

23. Olsen E, Laursen B, Vinzents PS. Bias and random errors in historical data of exposure to organic solvents. Am Ind Hyg Assoc J 1991;52:204-11.

24. Stewart PA, Zey JN, Hornung R, Herrick RF, Dosemeci M, Zaebst D, et al. Exposure assessment for a study of workers exposed to acrylonitrile, III: evaluation of exposure assessment methods. Appl Occup Environ Hyg 1996;1312-21.

25. Eisen EA, Smith TJ, Wegman DH, Louis TA, Froines J. Estimation of long term dust exposures in the Vermont granite sheds. Am Ind Hyg Assoc J 1984;45:89-94.

26. Woskie SR, Smith TJ, Hammond SK, Schenker MB, Garshick $\mathrm{E}$, Speizer FE. Estimation of the diesel exhaust exposures of railroad workers, II: national and historical exposures. Am J Ind Med 1988;13:395—404. 
27. Rice C, Harris RL, Lumsden JC, Symons MJ. Reconstruction of silica exposures in the North Carolina dusty trades. Am Ind Hyg Assoc J 1984;45:689-96.

28. Kalliokoski P. Estimating long-term exposure levels in process-type industries using production rates. Am Ind Hyg Assoc J $1990 ; 51: 310-2$

29. Dement JM, Harris RL, Symons MJ, Shy CM. Exposures and mortality among chrysotile asbestos workers, part I: exposure estimates. Am J Ind Med 1983;4:399 - 419.

30. Armstrong BG, Tremblay CG, Cyr D, Thériault GP. Estimating the relationship between exposure to tar volatiles and the incidence of bladder cancer in aluminum smelter workers. Scand J Work Environ Health 1986;12:486-93.

31. Breysse PN, Matanoski GM, Elliott EA, Francis M, Kaune W, Thomas K. 60 hertz magnetic field exposure assessment for an investigation of leukemia in telephone line workers. Am J Ind Med 1994;26:681-91.

32. Yu RC, Tan W-Y, Mathew RM, Andjelkovich DA, Levine RJ. A deterministic mathematical model for quantitative estimation of historical exposure. Am Ind Hyg Assoc J 1990;51: 194-201.

33. Stewart PA, Blair A, Cubit DA, Bales RE, Kaplan SA, Ward $\mathrm{J}$, et al. Estimating historical exposures to formaldehyde in a retrospective mortality study. Appl Ind Hyg 1986;1:34—41.

34. Esmen N. Retrospective industrial hygiene surveys. Am Ind Hyg Assoc J 1979;40:58-65.

35. Blair A, Stewart P, O'Berg M, Gaffey W, Walrath J, Ward J, et al. Mortality among industrial workers exposed to formal- dehyde. JNCI 1986;76:1071-84.

36. Zhao LP, Kolonel LN. Efficiency loss from categorizing quantitative exposures into qualitative exposures in case-control studies. Am J Epidemiol 1992;136:464-74.

37. Loomis DP, Peipins LA, Browning SR, Howard RL, Kromhout $\mathrm{H}$, Savitz DA. Organization and classification of work history data in industry-wide studies: an application to the electric power industry. Am J Ind Med 1994;226:413 - 25 .

38. Post W, Kromhout H, Heederik D, Noy D, Duijzentkunst RS. Semiquantitative estimates of exposure to methylene chloride and styrene: the influence of quantitative exposure data. Appl Occup Environ Hyg 1991;6:197—204.

39. Vinzents P, Carton B, Fjeldstad P, Rajan B, Stamm R. Comparison of exposure measurements stored in European databases on occupational air pollutants and definition of core information. Appl Occup Environ Hyg 1995;10:351-4.

40. Stewart PA, Lemanski D, White D, Zey J, Herrick RF, Masters $\mathrm{M}$, et al. Exposure assessment for a study of workers exposed to acrylonitrile, I: job exposure profiles: a computerized data management system. Appl Occup Environ Hyg 1992; 7:820-5.

41. Stewart PA, Triolo H, Zey J, White D, Herrick RF, Hornung $\mathrm{R}$, et al. Exposure assessment for a study of workers exposed to acrylonitrile, II: a computerized exposure assessment program. Appl Occup Environ Hyg 1995;10:698-706.

42. Dosemeci M, Wacholder S, Lubin JH. Does nondifferential misclassification of exposure always bias a true effect toward the null value? Am J Epidemiol 1990;132:746-8. 